\title{
A Multicenter, Randomized, Triple-Masked, Placebo-Controlled Trial of The Effect of Ambulatory Continuous Femoral Nerve Blocks on Discharge-Readiness Following Total Knee
} Arthroplasty In Patients on General Orthopaedic Wards

\author{
Brian M. Ilfeld, MD, MS [Associate Professor] ${ }^{1}$, Edward R. Mariano, MD, MAS [Associate \\ Clinical Professor] $^{2}$, Paul J. Girard, MD [Assistant Clinical Professor] ${ }^{3}$, Vanessa J. Loland, \\ MD [Assistant Clinical Professor] ${ }^{4}$, R. Scott Meyer, MD [Associate Clinical Professor] ${ }^{5}$, \\ John F. Donovan, MD [Assistant Clinical Professor] ${ }^{6}$, George A. Pugh, MD [Chair] ${ }^{7}$, Linda \\ T. Le, MD [Assistant Professor $]^{8}$, Daniel I. Sessler, MD [Professor and Chair ${ }^{9}$, Jonathan J. \\ Shuster, PhD [Research Professor] ${ }^{10}$, Douglas W. Theriaque, MS [Director of \\ Informatics] $^{11}$, and Scott T. Ball, MD [Assistant Clinical Professor] ${ }^{12}$ \\ ${ }^{1}$ Department of Anesthesiology, University of California San Diego, San Diego, California \\ 2 Department of Anesthesiology, University of California San Diego, San Diego, California \\ ${ }^{3}$ Department of Orthopaedic Surgery, University of California San Diego, San Diego, California \\ ${ }^{4}$ Department of Anesthesiology, University of California San Diego, San Diego, California \\ ${ }^{5}$ Department of Orthopaedic Surgery, Veteran's Affairs Medical Center, La Jolla, California \\ ${ }^{6}$ Department of Anesthesiology, University of California San Francisco, San Francisco, \\ California, Alta Bates Summit Medical Center, Oakland, California \\ 7 Department of Orthopaedics, Alta Bates Summit Medical Center, Oakland, California \\ 8 Departments of Anesthesiology, University of Florida, Gainesville, Florida \\ ${ }^{9}$ Department of Outcomes Research, Cleveland Clinic, Cleveland, Ohio \\ ${ }^{10}$ Department of Epidemiology and Health Policy Research, University of Florida, Gainesville, \\ Florida \\ ${ }^{11}$ General Clinical Research Center, University of Florida, Gainesville, Florida \\ ${ }^{12}$ Department of Orthopaedic Surgery, University of California San Diego, San Diego, California
}

\footnotetext{
Communicating Author: Brian M. Ilfeld, MD, MS (Clinical Investigation), UCSD Center for Pain Medicine, 9300 Campus Point Dr. -- MC 7651, La Jolla, California, United States 92037-7651. Telephone: (858) 543-5742; Fax: (603) 258-7782; bilfeld@ ucsd.edu.

Summary Statement: A four-day ambulatory continuous femoral nerve block decreases the time to reach three important discharge criteria by $20 \%$ following knee arthroplasty, primarily by improving analgesia.

Conflicts of Interest: Teleflex Medical and Stryker Instruments provided funding and donated portable infusion pumps for this investigation, these two companies had absolutely no input into any aspect of study conceptualization, design, and implementation; data collection, analysis and interpretation; or manuscript preparation. The funding from these companies was used by each institution to help defray research coordinator expenses. Drs. Mariano and Loland conduct continuous peripheral nerve block workshops for Stryker Instruments. No other author has a personal financial interest in this research.

Publisher's Disclaimer: This is a PDF file of an unedited manuscript that has been accepted for publication. As a service to our customers we are providing this early version of the manuscript. The manuscript will undergo copyediting, typesetting, and review of the resulting proof before it is published in its final citable form. Please note that during the production process errors may be discovered which could affect the content, and all legal disclaimers that apply to the journal pertain.
} 


\section{Abstract}

A continuous femoral nerve block (cFNB) involves the percutaneous insertion of a catheter adjacent to the femoral nerve, followed by a local anesthetic infusion, improving analgesia following total knee arthroplasty (TKA). Portable infusion pumps allow infusion continuation following hospital discharge, raising the possibility of decreasing hospitalization duration. We therefore used a multicenter, randomized, triple-masked, placebo-controlled study design to test the primary hypothesis that a four-day ambulatory cFNB decreases the time until each of three predefined readiness-for-discharge criteria (adequate analgesia, independence from intravenous opioids, and ambulation $\geq 30$ meters) are met following TKA compared with an overnight inpatient-only cFNB. Preoperatively, all patients received a cFNB with perineural ropivacaine $0.2 \%$ from surgery until the following morning, at which time they were randomized to either continue perineural ropivacaine $(n=39)$ or switch to normal saline $(n=38)$. Patients were discharged with their cFNB and portable infusion pump as early as postoperative day three. Patients given four days of perineural ropivacaine attained all three criteria in a median $\left(25^{\text {th }}-75^{\text {th }}\right.$ percentiles) of 47 (29-69) hours, compared with 62 (45-79) hours for those of the control group (Estimated ratio $=0.80,95 \%$ confidence interval: $0.66-1.00 ; \mathrm{p}=0.028)$. Compared with controls, patients randomized to ropivacaine met the discharge criterion for analgesia in $20(0-38)$ vs. 38 (15-64) hours ( $\mathrm{p}=0.009)$, and intravenous opioid independence in 21 (0-37) vs. 33 (11-50) hours $(\mathrm{p}=0.061)$. We conclude that a four-day ambulatory $\mathrm{cFNB}$ decreases the time to reach three important discharge criteria by an estimated $20 \%$ following TKA compared with an overnight cFNB, primarily by improving analgesia.

\section{Introduction}

While total knee arthroplasty (TKA) improves patients' long-term quality-of-life, it often results in severe pain in the immediate postoperative period that is difficult to control with solely oral and intravenous analgesics [36]. Adding a continuous femoral nerve block (cFNB) - also termed perineural local anesthetic infusion—is a relatively novel option. This technique involves the percutaneous insertion of a catheter directly adjacent to the femoral nerve below the inguinal ligament, followed by an infusion of dilute local anesthetic (Figure A, supplemental material). In hospitalized patients following TKA, addition of a cFNB to a multimodal analgesic regimen improves analgesia and accelerates passive knee flexion [7;36]. However, with few exceptions, perineural infusion benefits are provided only during the infusion itself and cease when the perineural catheter is removed, negating the possibility of shortening hospitalization duration [33]. As a result, practitioners are left with the perplexing choice of requiring an extended hospitalization to provide superior analgesia and other infusion benefits, or allowing earlier hospital discharge with potentially inadequate analgesia and suboptimal rehabilitation [33].

Unlike intravenous or epidural analgesics, perineural infusion may be continued following hospital discharge using a portable infusion pump to provide local anesthetic on an ambulatory basis [16]. Ambulatory cFNB thus offers the theoretic potential of providing prolonged analgesia while simultaneously decreasing disability and hospitalization duration [17]. A previous investigation reported a four-day ambulatory cFNB decreased the time required to reach three important discharge criteria by 53\% compared with an overnight, hospital-only cFNB following TKA $(\mathrm{p}<0.001)$ [18]. However, a major limitation of that study was the highly artificial research environment: (1) all subjects convalesced in a specialized, stand-alone clinical research center often with a single nurse for each patient; (2) a single anesthesiologist cared for all subjects; and (3) the three physical therapists involved in the study were specially trained to optimize the study protocol, including ambulation distances [18]. Consequently, it is problematic generalizing results from this 
single trial to the more than half-million patients who undergo knee arthroplasty each year in the United States [25], much less in the rest of the world.

We therefore designed this prospective clinical trial to test the hypothesis that an extended ambulatory cFNB shortens the time until three specific, predefined readiness-for-discharge criteria are met following TKA; and to allow inference of the results to the general population by using standard orthopaedic hospital wards in multiple centers with a wide range of healthcare providers (e.g., surgeons, anesthesiologists, nurses, physical therapists, etc). The three discharge criteria for this multicenter, randomized, triple-masked (patients, investigators, and statisticians), placebo-controlled investigation included (1) adequate analgesia; (2) independence from intravenous opioids; and (3) sufficient ambulation to allow home discharge. These criteria were chosen because failure to meet one or more of them accounts for the majority of hospitalization days at many institutions.

\section{Materials and Methods}

\section{Enrollment}

This study adhered to the guidelines of the Committee of Research and Ethical Issues of IASP. The Institutional Review Board at each participating clinical center approved all study procedures and the trial was prospectively registered at clinicaltrials.gov (NCT00419276). All subjects provided written, informed consent; and because this was a multi-center trial, a Data Safety Monitoring Board (University of California San Diego, San Diego, California) reviewed data and adverse events every six months. Subjects offered enrollment included adults (18-75 years) scheduled for primary, unilateral, tricompartment, cemented TKA via a $12-18 \mathrm{~cm}$ midline skin incision and parapatellar approach who desired a cFNB for postoperative analgesia. Exclusion criteria included a history of alcohol or opioid dependence, current chronic analgesic therapy (daily use $>4$ weeks), allergy to study medications, known renal insufficiency (creatinine $>1.5 \mathrm{mg} / \mathrm{dL}$ ), morbid obesity (body mass index $>40 \mathrm{~kg} / \mathrm{m}^{2}$ ), pregnancy, incarceration, or comorbidity that resulted in moderateto-severe functional limitation (American Society of Anesthesiologists physical status classification > 2) [32].

\section{Perioperative procedures}

A femoral perineural catheter (StimuCath, Teleflex Medical, Research Triangle Park, North Carolina) was inserted in all subjects using a nerve stimulator (Stimuplex-DIG; B. Braun Medical, Bethlehem, PA) initially set at $1.2 \mathrm{~mA}, 0.1 \mathrm{~ms}$, and $2 \mathrm{~Hz}$, using a technique similar to one previously described with a muscle contraction end-point of the quadriceps at 0.20 $0.50 \mathrm{~mA}$ via the placement needle (Figure 1) [15;21]. Forty milliliters of mepivacaine $1.5 \%$, with epinephrine, $100 \mu \mathrm{g}$, was injected via the catheter with gentle aspiration every $3 \mathrm{~mL}$. The femoral nerve block was evaluated 20 minutes later and considered successful when subjects were unable to extend the knee and experienced a decreased sensation to cold temperature in the femoral nerve distribution. Subjects with a successful catheter placement and nerve block onset per protocol were retained in the study.

For the surgical procedure, subjects were given a volatile anesthetic in nitrous oxide and oxygen. A ropivacaine $0.2 \%$ infusion was initiated via the femoral catheter with a basal rate of $6 \mathrm{~mL} / \mathrm{h}$, patient-controlled bolus-dose of $4 \mathrm{~mL}$, and lock-out period of $30 \mathrm{~min}$.

All subjects received the ropivacaine perineural infusion initiated in the operating room and continued until the morning after surgery, as well as one week of oral acetaminophen (975 $\mathrm{mg}$ four times daily), a sustained-release oral opioid (Oxycontin, $10 \mathrm{mg}$ every $12 \mathrm{~h}$ ); and celecoxib (200 mg every $12 \mathrm{~h}$ ). For breakthrough pain, subjects were instructed to depress the bolus button on their infusion pump and wait at least 15 min for analgesic onset. Rescue 
opioid was determined by pain severity using a Numeric Rating Scale (NRS) of 0-10 [9], with 0 equal to no pain and 10 being the worst imaginable pain: oxycodone $5 \mathrm{mg}$ (NRS < 4) or $10 \mathrm{mg}$ (NRS $\geq 4$ ). Pain was reassessed $30 \mathrm{~min}$ later and intravenous morphine ( $2-4 \mathrm{mg}$ ) was provided until the NRS $<4$.

\section{Randomization and intervention}

Subject demographic and catheter placement data were uploaded via the Internet to a secure [40], password-protected, encrypted central server (www.PAINfRE.com, General Clinical Research Center, Gainesville, Florida) [2]. Subjects were allocated to treatment only after confirmation of a successful initial surgical block preoperatively. Subjects were randomized to one of two groups-ropivacaine $0.2 \%$ or normal saline (placebo) — stratified by institution/hospital using computer-generated tables and provided to investigational pharmacists via the PAINfRE.com Web site. An investigational pharmacist at each institution prepared all study solution as determined by these lists. Ropivacaine and normal saline are indistinguishable in appearance, and therefore investigators, subjects, and all clinical staff were masked to treatment group assignment. Unmasking did not occur until statistical analysis was complete (termed "triple masking"). At 06:00 on postoperative day (POD) 1, each patient's infusion pump which contained ropivacaine $0.2 \%$ was replaced with an infusion pump filled with study solution containing either additional ropivacaine $0.2 \%$ or normal saline (Figure B, supplemental material).

Pain scores were recorded every four hours (except when subjects were sleeping) and when subjects requested analgesics. Subjects underwent physical therapy twice daily beginning the morning following surgery and thereafter until discharge (Figure 2). If the physical therapist believed subject ambulation was limited due to quadriceps weakness, the perineural infusion was stopped for one hour and then restarted at half the previous basal rate. At 18:00 on POD 2 (36 h following randomization), a portable infusion pump (Pain Pump 2 Blockaid, Stryker Instruments, Kalamazoo, MI) containing $400 \mathrm{~mL}$ of the same study solution replaced the previous infusion pump (basal $5 \mathrm{~mL} / \mathrm{h}$ regardless of previous basal rate; bolus $4 \mathrm{~mL}$; lock-out $60 \mathrm{~min}$ ).

\section{Outcome Measurements}

Staff masked to treatment group assignment performed all measures and assessments.

At our institutions, failure to meet three criteria account for the majority of hospitalization days. These three criteria include (1) adequate analgesia (defined as pain less than 4 on a NRS of 0-10) [4]; (2) independence from intravenous opioids; and (3) sufficient ambulation of at least $30 \mathrm{~m}$ without time limit [10]. Therefore, the primary end point for this study was the time from surgical stop until all three of these criteria were fulfilled - without a reversion to unfulfilled status. Fulfillment of criteria was evaluated at the end of each eighthour nursing shift.

Major secondary endpoints included pain scores as measured on a NRS, supplemental opioid consumption, attaining a standing position without assistance, ambulation distance, and passive knee flexion and extension. Range-of-motion was measured using a goniometer prior to ambulation with the subject in the supine position during each physical therapy session. Knee immobilizers were not used.

Subjects were discharged home or to a rehabilitation center with their portable infusion pump and perineural catheter in situ. Subjects were discharged after meeting the three main discharge criteria at the discretion of their orthopaedic surgeon, but never prior to the morning of POD 3. Subjects and their caretakers were provided with verbal and written catheter/pump instructions, the telephone and pager numbers of an investigator available at 
all times, and prescriptions for their outpatient oral medications that did not differ from the oral analgesics provided in the hospital. Subjects were telephoned in the evenings through POD 5. In the evening of POD 4, subjects' caretakers removed the femoral catheters with physician instructions provided by telephone.

\section{Statistical methods}

The study was powered for the primary end point. Based on a pilot study [17], the planning distribution for time-to-discharge readiness for the Ropivacaine (Placebo) group was $30 \mathrm{~h}$ : 60\% (25\%); 45 h: 20\% (25\%); 54 h: 10\% (25\%); and 69+ h: 10\% (25\%). To ensure 90\% power at $\mathrm{P}=0.05$ (two-sided) for the Wilcoxon Rank Sum Test, 38 subjects were required in each treatment group on the basis of the formula of Shuster, Chang, and Tian [34]. To allow for a higher variability in the primary end point, we planned to enroll a total of 80 subjects. The actual study power is somewhat higher as better granularity of the actual discharge qualification times occurred, making the outcome more continuous. In practice, we used the actual times and not the grouped times.

For consistency, all outcome variables (primary and secondary) were analyzed by the Twosided Wilcoxon Rank Sum Test, which provides distribution-free $P$-values and is highly robust against outliers. For the primary variable, effect size was estimated by the method of Hodges and Lehmann for a scale parameter [12;35]. For convenient nomenclature, a twosided $P<0.05$ was considered significant. Because each comparison dilutes all other Pvalues, we restricted our analysis to 11 comparisons among secondary end points [27]. Post hoc comparison of the incidence of subject falls between the two treatment groups was made using the Fisher's Exact test. Significant findings in secondary outcomes should be viewed as suggestive, requiring confirmation in a future trial before considering them as definitive [27].

\section{Results}

During a 29-month period between April 2007 and August 2009, 81 subjects enrolled and all but one $(99 \%)$ had a perineural catheter successfully positioned per protocol (Supplemental Figure C; Table 1). Of these subjects, 40 were randomized to be switched from perineural ropivacaine to normal saline (Placebo group) at 06:00 on POD 1, while 40 continued to receive perineural ropivacaine through POD 4 (Ropivacaine group, Figure 2). One subject in the Placebo group had an infection of his knee joint discovered in the operating room and his procedure cancelled. Similarly, one subject from the Ropivacaine group had his procedure canceled for medical reasons following catheter insertion. And one final subject from the Placebo group underwent a rule-out myocardial infarction immediately following surgery due to electrocardiogram changes (no evidence of infarction was detected). All three of these individuals requested study withdrawal, and subsequent data was excluded from analysis, as mandated by U.S. ethical guidelines [1]. Therefore, 77 subjects were included in the analysis.

\section{Primary end point}

Subjects in the Ropivacaine group attained all three discharge criteria in a median $\left(25^{\text {th }}-75^{\text {th }}\right.$ percentiles) of 47 (29-69) hours, compared with 62 (45-79) hours for those of the Placebo group (Figure 1; Estimated ratio $=0.80,95 \%$ confidence interval: $0.66-1.00 ; \mathrm{p}=0.028$ ).

\section{Secondary end points}

While the between-group differences in reported pain and intravenous morphine requirements were relatively small, a higher percentage of subjects receiving ropivacaine met discharge criteria at each post-randomization time-point (Figures 2 and 3). In contrast, 
the two groups were nearly identical for distance of ambulation (Figure 4), knee range-ofmotion (Figure 5), and ability to stand unassisted (Table 2). Ten subjects (26\%) of the Ropivacaine group had their basal ropivacaine infusion halved on POD 1 because of quadriceps weakness versus 9 subjects (24\%) of the Placebo group. Three of the ten subjects in the Ropivacaine group ( $8 \%$ of total group) required a second halving of their basal rate because of continued quadriceps weakness. Hospitalization duration was a median (range) of 3.0 (3.0-5.0) days in the Ropivacaine group and 3.5 (3.0-12.0) days in the Placebo group $(p=0.098)$.

\section{Protocol violations and adverse events}

Eight subjects—-four in each treatment group — had their perineural catheters inadvertently dislodged prior to POD 4. Catheter dislodgement is a well-known (and unfortunately somewhat common) issue during perineural infusion and does not result in patient injury. For purposes of analysis, each of these subjects was retained in their respective treatment group per the intention-to-treat principle [39].

From the Placebo group, two subjects developed thrombocytopenia on POD 2 (one with a history of mild Von Willebrand disease). Their low molecular weight heparin and celebrex were discontinued and both received transfusions raising platelet counts above 100,000, allowing home discharge on POD 4 and 11 following perineural catheter removal without incident. From the Ropivacaine group, one subject required treatment for evolving delirium tremens on POD 2 and at that time acknowledged that he was dependent upon a high daily alcohol intake — an exclusion criterion of this study — and the subject requested study withdrawal. Three additional subjects from the Ropivacaine group fell during their perineural infusions: one the evening of POD 0 while sitting; one while trying to stand POD 2 without assistance; and one while exiting her car after arriving home following discharge on POD 3 as well as the following day walking her dog. The first subject sustained a small abrasion above her left eye while the other two subjects were uninjured. Of note, there were no femoral nerve palsies reported the day following catheter removal (POD 5) or at the sixweek surgical postoperative visit [11].

\section{Discussion}

This multicenter investigation provides evidence that for subjects convalescing in a typical orthopaedic ward following TKA, extending a cFNB from overnight to over four days decreases the time to reach each of three important discharge criteria by an estimated $20 \%$. Furthermore, hospitalization for the duration of the infusion is not required when a portable pump is used, and subjects or their caretakers remove the perineural catheters following discharge. Our results thus suggest that by primarily decreasing postoperative pain, the duration of hospitalization following TKA may be shortened in some cases while still providing the benefits of cFNB.

\section{Inference of results}

Ambulatory cFNB following TKA has been studied previously, but in the highly artificial environment of a stand-alone clinical research center [18]. In addition, a single anesthesiologist provided all cFNB-related care from catheter insertion through follow-up after catheter removal [18]. Within this environment, subjects with a four-day cFNB met three discharge criteria in a median $\left(25^{\text {th }}-75^{\text {th }}\right.$ percentiles $)$ of 25 (21-47) hours, compared with 71 (46-89) hours for those of the control group with an overnight, hospital-only infusion. Providing the extended cFNB thus resulted in a far greater decrease in time until discharge readiness (46 hours, or 53\%; $\mathrm{p}<0.001$ ) than found for subjects of the current study ( 15 hours, or $20 \%$; $\mathrm{p}=0.028$ ). That the benefit was greater in a highly-controlled 
environment is hardly surprising. Our current results reflect a country-wide diversity of practice that is probably more representative of what clinicians can generally expect from an extended, ambulatory cFNB.

Our study protocol provided supplemental analgesics as necessary until pain was acceptably low (NRS < 4); it is thus unsurprising there was little difference in pain scores between the two treatment groups at each time point in both investigations [18]. However, in the previously-published study, subjects receiving an extended cFNB achieved independence from intravenous opioids 19 hours sooner (medians of 44 versus 25 hours; $p=0.044$ ), compared with only 12 hours in the current study (33 versus 21 hours; $p=0.061$ ). This difference most likely resulted from the fact that subjects in the current study convalesced on standard orthopaedic wards where 1:6 to 1:8 nurse-patient ratios were the norm, whereas it was usually 1:1 ratio in the previously-published study. With a 1:1 ratio, nursing staff are naturally far more available to provide intravenous opioids for pain spikes, as is commonly needed for postoperative pain which often waxes and wanes.

\section{Ambulation}

In contrast, there was less between-group improvement for either study in the time until ambulating 30 meters by extending the cFNB ( 5 hours in the current study, $\mathrm{p}=0.780$ ); however, the time until reaching this criterion between treatment groups for the extended and overnight cFNB in the previous study was 23 and 31 hours, respectively, compared with 40 and 45 hours of the current investigation. Again, we can only speculate as to the reason(s) why the subjects in both treatment groups of the previous study ambulated at least 30 meters so much earlier than the current study; but it is likely due, in part, to the physical therapists involved. In the previous study, only three physical therapists specifically trained to maximize ambulatory distance were involved in patient care; while in the current investigation there were many physical therapists spread over multiple institutions who were asked to have subjects ambulate to the best of their ability, but no specific study-related instructions given beyond that one request. As with the time until intravenous opioid independence, it is probable that the ambulation results of the current investigation are far more indicative of what practitioners can expect for their own subjects by providing an extended, ambulatory cFNB following TKA.

Many believe that pain limits ambulation after arthroplasty [22]. However, although analgesia was improved in our extended infusion subjects, it did not result in increased ambulation distance. One explanation for this lack of improvement is that a well-recognized side effect of perineural infusion is muscular weakness [5], particularly undesirable in cFNB that affect quadriceps femoris function required for ambulation. Because an extended cFNB was not correlated with a decrease in ambulation distance as well, it is possible that the disadvantage of muscle weakness is offset by improved analgesia. However, a weakened quadriceps femoris muscle is a risk factor for falling in elderly patients, regardless of analgesia [38]. In the current study, two subjects fell (twice in one subject for a total of 3 falls), and both were in the Ropivacaine group ( $\mathrm{p}=0.240)$. There is growing evidence that lower extremity continuous peripheral nerve blocks increase the risk of patient falls $[11 ; 14 ; 18 ; 29 ; 31 ; 41]$. Whether or not the cFNB was a contributing factor to the falls of the present study remains unknown because our investigation was not powered to detect such (presumably) rare complications [24]. Nonetheless, patient falls during perineural infusion are now being highlighted in the anesthesiology and surgical literature [11;23;29].

\section{Fall prevention}

Until additional data are available, practitioners may want to consider steps that may minimize the risk of falls. Such steps include minimizing the dose of local anesthetic; 
providing limited-volume patient-controlled bolus doses which allow for a decreased basal dose without compromising analgesia in some cases [8;19]—although not all [20]; utilizing a knee immobilizer and walker/crutches during ambulation [29]; and educating all healthcare providers of possible continuous peripheral nerve block-induced muscle weakness and necessary fall precautions. Of note, a similar percentage of subjects in each treatment group ( $26 \%$ vs. $24 \%$ ) had a decrease in their basal infusion rate when quadriceps femoris weakness limited ambulation, suggesting that the muscle weakness was not due to the perineural ropivacaine infusion. However, three of the ten subjects of the Ropivacaine group required a second halving of their basal rate because of continued quadriceps weakness, versus none of the Placebo group subjects. This association demonstrates the importance of a using an infusion pump that allows the basal rate to be adjusted during the infusion. The optimal basal infusion rate and ropivacaine concentration remain undetermined following TKA; and initially providing a lower rate or concentration may result in decreased cFNB benefits for a subset of patients [6;37].

\section{Study Limitations}

The control (Placebo) group received an initial femoral nerve block followed by an overnight cFNB, and not simply a single-injection femoral nerve block and/or opioids as is common in many practices in the United States. Additionally, although all subjects received sustained-release oral opioids, these may result in undesirable side effects which were not assessed in this investigation. This study also excluded patients who had taken opioids daily for more than the previous four weeks. Although precise figures are unavailable, undoubtedly a large percentage of patients undergoing TKA have received over a month of opioids daily, and whether the results of the current study remain applicable to this patient subset remains unknown.

\section{Early discharge}

Our study protocol required subjects to remain hospitalized until at least POD 3, even if they were discharge-ready earlier. This partially explains why subjects of the Ropivacaine group had a median (range) actual hospitalization duration of 3 (3-5) days even though they met the three main discharge criteria in a median of less than 2 days. Furthermore, there is usually a substantial difference between discharge-readiness and actual discharge because additional factors such as patient comorbidities [28], rehabilitation expectations [7;36], and hospital policy $[13 ; 30]$ may significantly influence hospitalization duration. While this investigation suggests that the duration of hospitalization after TKA may be decreased with ambulatory cFNB, it does not define an appropriate subset of patients and incidence of complications associated with early discharge. Caution is warranted because, following TKA, the median times to myocardial infarction and pulmonary embolism are one and four days, respectively [26].

Although the feasibility of converting TKA into an overnight-stay procedure using ambulatory cFNB has been previously demonstrated,[17] a single series of patients does not permit conclusions to be drawn regarding the relative safety of this practice. Therefore, additional study is required to define an appropriate subset of patients and assess the incidence of complications associated with earlier discharge following TKA. Nonetheless, ambulatory cFNB may enable rapid hospital discharge to a skilled nursing facility or rehabilitation center where medical oversight would continue in case of an adverse event [3].

\section{Supplementary Material}

Refer to Web version on PubMed Central for supplementary material. 


\section{Acknowledgments}

The authors gratefully acknowledge the invaluable assistance of Thomas Peatman, MD, Attending Physician, Department of Orthopedics, Alta Bates Summit Medical Center (Oakland, California); Beverly Morris, RN, CNP, $M B A$, Educator, Department of Nursing, University of California San Diego (San Diego, California); Patrick Olsen, $R N, B S N$, Nurse Manager, and Claire Jenkins, RN, Assistant Nurse Manager, Hillcrest Hospital (San Diego, California); Eliza Ferguson, BS, Research Coordinator, Department of Anesthesiology, University of California San Diego (San Diego, California); and Vickie Nolan, RN, CCRC, Clinical Research Manager, Jordan Research and Education Institute, Alta Bates Summit Medical Center (Oakland, California).

Funding Sources: Funding for this project was provided by the National Institutes of Health grant GM077026 from the National Institute of General Medical Sciences (Bethesda, Maryland); National Institutes of Health grants RR00082, RR000827, and RR025208 from the National Center for Research Resources (Bethesda, Maryland); the Department of Anesthesiology, University of California San Diego (San Diego, California); Stryker Instruments (Kalamazoo, Michigan); and Teleflex Medical (Research Triangle Park, North Carolina). The contents of this article are solely the responsibility of the authors and do not necessarily represent the official views of these entities.

\section{Reference List}

1. The Common Rule, Title 45 (Public Welfare), Code of Federal Regulations, Part 46 (Protection of Human Subjects). U.S. Department of Health and Human Services, National Institutes of Health, and Office for Human Research Protections; 2001. p. 1-18.

2. Avidan A, Weissman C, Sprung CL. An internet web site as a data collection platform for multicenter research. Anesth Analg 2005;100:506-11. [PubMed: 15673884]

3. Bickler P, Brandes J, Lee M, Bozic K, Chesbro B, Claassen J. Bleeding complications from femoral and sciatic nerve catheters in patients receiving low molecular weight heparin. Anesth Analg 2006;103:1036-1037. [PubMed: 17000826]

4. Bijur PE, Latimer CT, Gallagher EJ. Validation of a verbally administered numerical rating scale of acute pain for use in the emergency department. Acad Emerg Med 2003;10:390-392. [PubMed: 12670856]

5. Borgeat A, Kalberer F, Jacob H, Ruetsch YA, Gerber C. Patient-controlled interscalene analgesia with ropivacaine $0.2 \%$ versus bupivacaine $0.15 \%$ after major open shoulder surgery: The effects on hand motor function. Anesth Analg 2001;92:218-223. [PubMed: 11133631]

6. Brodner G, Buerkle H, Van Aken H, Lambert R, Schweppe-Hartenauer ML, Wempe C, Gogarten W. Postoperative analgesia after knee surgery: A comparison of three different concentrations of ropivacaine for continuous femoral nerve blockade. Anesth Analg 2007;105:256-262. [PubMed: 17578984]

7. Capdevila X, Barthelet Y, Biboulet P, Ryckwaert Y, Rubenovitch J, d'Athis F. Effects of perioperative analgesic technique on the surgical outcome and duration of rehabilitation after major knee surgery. Anesthesiology 1999;91:8-15. [PubMed: 10422923]

8. Capdevila X, Dadure C, Bringuier S, Bernard N, Biboulet P, Gaertner E, Macaire P. Effect of patient-controlled perineural analgesia on rehabilitation and pain after ambulatory orthopedic surgery: A multicenter randomized trial. Anesthesiology 2006;105:566-573. [PubMed: 16931991]

9. Cepeda MS, Africano JM, Polo R, Alcala R, Carr DB. What decline in pain intensity is meaningful to patients with acute pain? Pain 2003;105:151-157. [PubMed: 14499431]

10. Enloe LJ, Shields RK, Smith K, Leo K, Miller B. Total hip and knee replacement treatment programs: a report using consensus. J Orthop Sports Phys Ther 1996;23:3-11. [PubMed: 8749744]

11. Feibel RJ, Dervin GF, Kim PR, Beaule PE. Major complications associated with femoral nerve catheters for knee arthroplasty: a word of caution. J Arthroplasty 2009;24:132-137. [PubMed: 19553071]

12. Hodges JL, Lehmann EL. Estimates of location based on rank tests. Ann Math Statist 1963;34:598-611.

13. Hughes K, Kuffner L, Dean B. Effect of weekend physical therapy treatment on postoperative length of stay following total hip and total knee arthroplasty. Physiother Can 1993;45:245-249. [PubMed: 10130908] 
14. Ilfeld BM, Ball ST, Gearen PF, Le LT, Mariano ER, Vandenborne K, Duncan PW, Sessler DI, Enneking FK, Shuster JJ, Theriaque DW, Meyer RS. Ambulatory continuous posterior lumbar plexus nerve blocks after hip arthroplasty: a dual-center, randomized, triple-masked, placebocontrolled trial. Anesthesiology 2008;109:491-501. [PubMed: 18719448]

15. Ilfeld BM, Enneking FK. Perineural catheter placement for a continuous nerve block: a single operator technique. Reg Anesth Pain Med 2003;28:154-155. [PubMed: 12677635]

16. Ilfeld BM, Enneking FK. Continuous peripheral nerve blocks at home: A review. Anesth Analg 2005;100:1822-1833. [PubMed: 15920221]

17. Ilfeld BM, Gearen PF, Enneking FK, Berry LF, Spadoni EH, George SZ, Vandenborne K. Total knee arthroplasty as an overnight-stay procedure using continuous femoral nerve blocks at home: A prospective feasibility study. Anesth Analg 2006;102:87-90. [PubMed: 16368810]

18. Ilfeld BM, Le LT, Meyer RS, Mariano ER, Vandenborne K, Duncan PW, Sessler DI, Enneking FK, Shuster JJ, Theriaque DW, Berry LF, Spadoni EH, Gearen PF. Ambulatory continuous femoral nerve blocks decrease time to discharge readiness after tricompartment total knee arthroplasty: a randomized, triple-masked, placebo-controlled study. Anesthesiology 2008;108:703-713. [PubMed: 18362603]

19. Ilfeld BM, Morey TE, Enneking FK. Infraclavicular perineural local anesthetic infusion: a comparison of three dosing regimens for postoperative analgesia. Anesthesiology 2004;100:395402. [PubMed: 14739817]

20. Ilfeld BM, Morey TE, Wright TW, Chidgey LK, Enneking FK. Interscalene perineural ropivacaine infusion: a comparison of two dosing regimens for postoperative analgesia. Reg Anesth Pain Med 2004;29:9-16. [PubMed: 14727272]

21. Ilfeld BM, Thannikary LJ, Morey TE, Vander Griend RA, Enneking FK. Popliteal sciatic perineural local anesthetic infusion: a comparison of three dosing regimens for postoperative analgesia. Anesthesiology 2004;101:970-977. [PubMed: 15448531]

22. Ilfeld BM, Vandenborne K, Duncan PW, Sessler DI, Enneking FK, Shuster JJ, Theriaque DW, Chmielewski TL, Spadoni EH, Wright TW. Ambulatory continuous interscalene nerve blocks decrease the time to discharge readiness after total shoulder arthroplasty: A randomized, triplemasked, placebo-controlled study. Anesthesiology 2006;105:999-1007. [PubMed: 17065895]

23. Kandasami M, Kinninmonth AW, Sarungi M, Baines J, Scott NB. Femoral nerve block for total knee replacement - a word of caution. Knee 2009;16:98-100. [PubMed: 19046884]

24. Klein SM, Nielsen KC, Greengrass RA, Warner DS, Martin A, Steele SM. Ambulatory discharge after long-acting peripheral nerve blockade: 2382 blocks with ropivacaine. Anesth Analg 2002;94:65-70. [PubMed: 11772802]

25. Kurtz S, Mowat F, Ong K, Chan N, Lau E, Halpern M. Prevalence of primary and revision total hip and knee arthroplasty in the United States from 1990 through 2002. J Bone Joint Surg Am 2005;87:1487-1497. [PubMed: 15995115]

26. Mantilla CB, Horlocker TT, Schroeder DR, Berry DJ, Brown DL. Frequency of myocardial infarction, pulmonary embolism, deep venous thrombosis, and death following primary hip or knee arthroplasty. Anesthesiology 2002;96:1140-1146. [PubMed: 11981154]

27. Mariano ER, Ilfeld BM, Neal JM. "Going fishing”-the practice of reporting secondary outcomes as separate studies. Reg Anesth Pain Med 2007;32:183-185. [PubMed: 17543811]

28. Munin MC, Kwoh CK, Glynn N, Crossett L, Rubash HE. Predicting discharge outcome after elective hip and knee arthroplasty. Am J Phys Med Rehabil 1995;74:294-301. [PubMed: 7632386]

29. Muraskin SI, Conrad B, Zheng N, Morey TE, Enneking FK. Falls associated with lower-extremitynerve blocks: a pilot investigation of mechanisms. Reg Anesth Pain Med 2007;32:67-72. [PubMed: 17196495]

30. Oldmeadow LB, McBurney H, Robertson VJ. Hospital stay and discharge outcomes after knee arthroplasty: implications for physiotherapy practice. Aust J Physiother 2002;48:117-121. [PubMed: 12047209]

31. Rodriguez J, Taboada M, Carceller J, Lagunilla J, Barcena M, Alvarez J. Stimulating popliteal catheters for postoperative analgesia after hallux valgus repair. Anesth Analg 2006;102:258-262. [PubMed: 16368839] 
32. Rozner MA. The American Society of Anesthesiologists physical status score and risk of perioperative infection. JAMA 1996;275:1544. [PubMed: 8622238]

33. Salinas FV, Liu SS, Mulroy MF. The effect of single-injection femoral nerve block versus continuous femoral nerve block after total knee arthroplasty on hospital length of stay and longterm functional recovery within an established clinical pathway. Anesth Analg 2006;102:12341239. [PubMed: 16551930]

34. Shuster JJ, Chang M, Tian L. Design of group sequential trials with ordinal categorical data based on the Mann-Whitney-Wilcoxon test. Sequential Analysis 2004;23:413-26.

35. Shuster JJ, Theriaque DW, Ilfeld BM. Applying Hodges-Lehmann scale parameter estimates to hospital discharge times. Clin Trials 2008;5:631-634. [PubMed: 19029215]

36. Singelyn FJ, Deyaert M, Joris D, Pendeville E, Gouverneur JM. Effects of intravenous patientcontrolled analgesia with morphine, continuous epidural analgesia, and continuous three-in-one block on postoperative pain and knee rehabilitation after unilateral total knee arthroplasty. Anesth Analg 1998;87:88-92. [PubMed: 9661552]

37. Singelyn FJ, Gouverneur JM. Extended "three-in-one" block after total knee arthroplasty: continuous versus patient-controlled techniques. Anesth Analg 2000;91:176-180. [PubMed: 10866908]

38. Stevens JE, Mizner RL, Snyder-Mackler L. Quadriceps strength and volitional activation before and after total knee arthroplasty for osteoarthritis. J Orthop Res 2003;21:775-779. [PubMed: 12919862]

39. Todd MM. Clinical research manuscripts in anesthesiology. Anesthesiology 2001;95:1051-1053. [PubMed: 11684970]

40. van Oostrom JH. Web-based data collection: security is only as good as the weakest link. Anesth Analg 2005;101:1888. [PubMed: 16301289]

41. Williams BA, Kentor ML, Bottegal MT. The incidence of falls at home in patients with perineural femoral catheters: a retrospective summary of a randomized clinical trial. Anesth Analg 2007;104:1002. [PubMed: 17377133] 


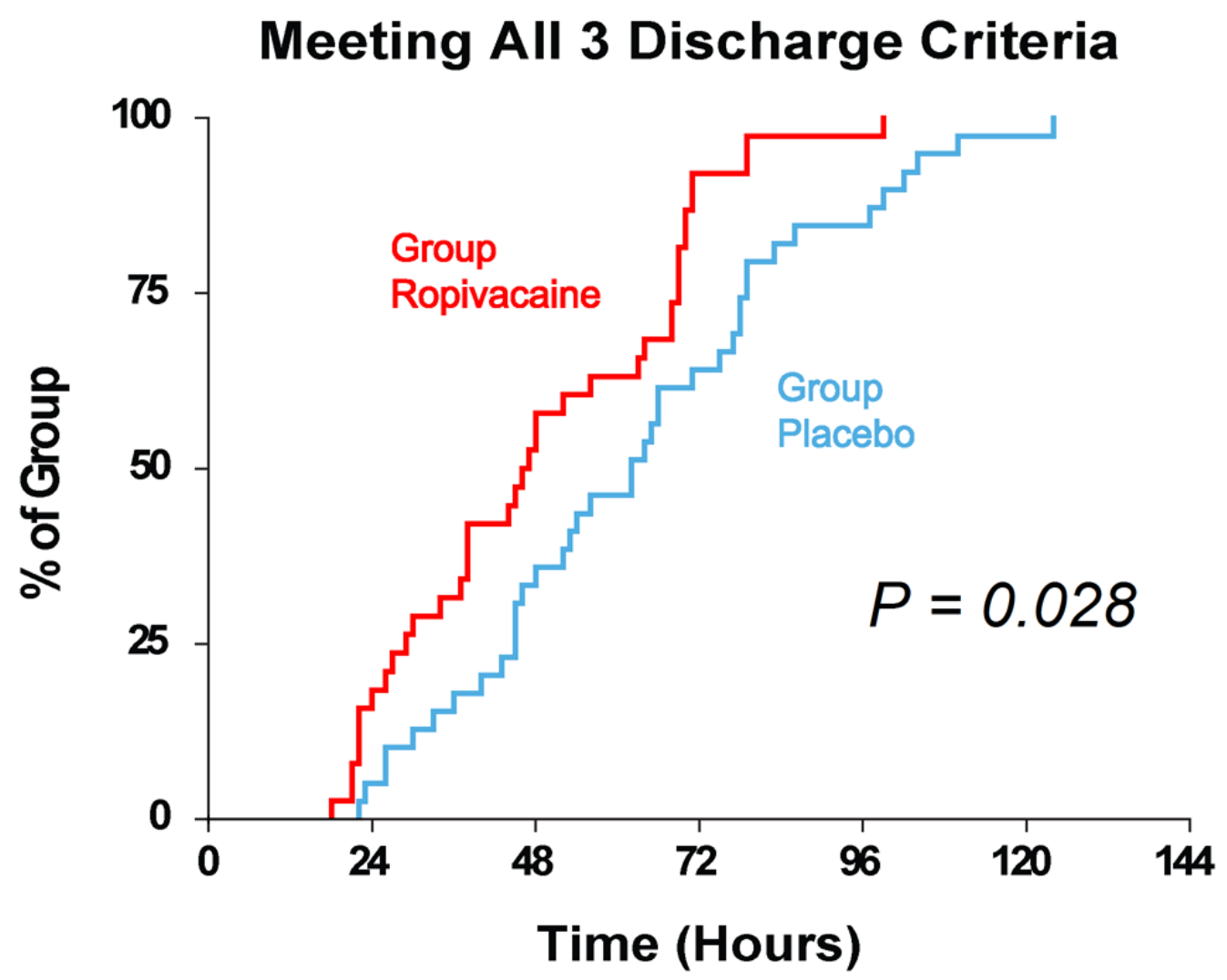

Figure 1.

Effect of femoral perineural ropivacaine infusion on the time to reach three important discharge criteria (adequate analgesia, independence from intravenous opioids, and the ability to ambulate at least $30 \mathrm{~m}$ ) following total knee arthroplasty. Data presented are Kaplan-Meier estimates of the cumulative percentages of subjects meeting all three discharge criteria at each time point and subsequent time points. Data are for subjects randomly assigned to group Ropivacaine (perineural ropivacaine from surgery through postoperative day 4) or group Placebo (perineural ropivacaine from surgery through 06:00 postoperative day 1 followed by perineural normal saline through postoperative day 4). Subjects given four days of perineural ropivacaine attained all 3 discharge criteria in a median $\left(25^{\text {th }}-75^{\text {th }}\right.$ percentiles) of 47 (29-69) hours, compared with 62 (45-79) hours for those of the control group (Estimated ratio $=0.80,95 \%$ confidence interval: 0.66-1.00; $\mathrm{p}=0.028$ ). 

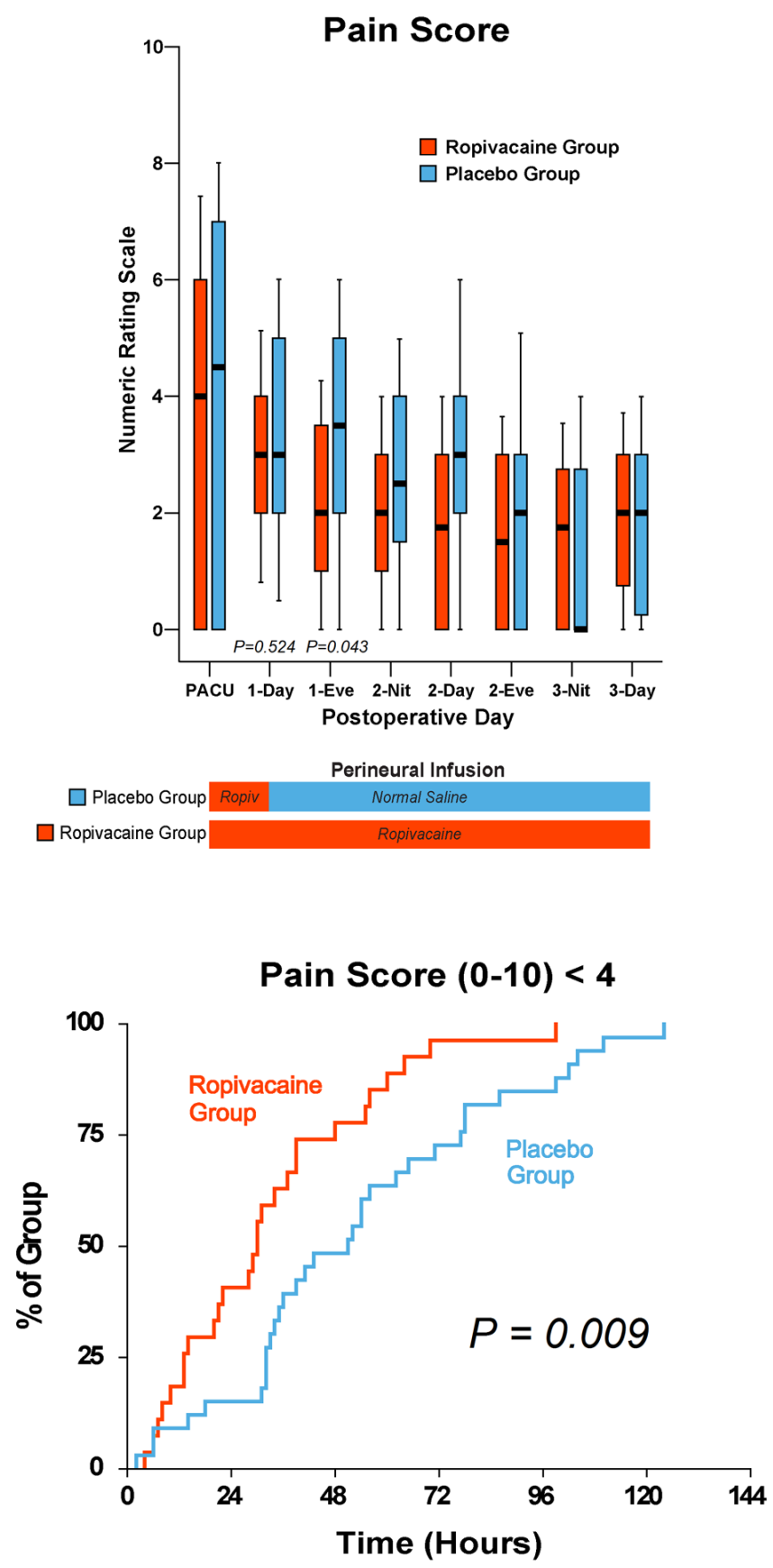

Figure 2.

Effects of femoral perineural ropivacaine infusion on postoperative pain following total knee arthroplasty. Pain severity indicated using a Numeric Rating Scale of 0-10, with 0 equal to no pain and 10 being the worst imaginable pain. Data are expressed as median (horizontal bar) with $25^{\text {th }}-75^{\text {th }}$ (box) and $10^{\text {th }}-90^{\text {th }}$ (whiskers) percentiles for subjects randomly assigned to group Ropivacaine (perineural ropivacaine from surgery through postoperative day 4) or group Placebo (perineural ropivacaine from surgery through 06:00 postoperative day 1 followed by perineural normal saline through postoperative day 4 ). Because each comparison dilutes all other P-values, we restricted our analysis to 11 comparisons among secondary end points. P-values are provided where statistical 
comparisons were applied. Kaplan-Meier estimates include the cumulative percentages of subjects with the discharge criterion of a median Numeric Rating Scale $<4$ in the 8-hour period and subsequent time points. Subjects given four days of perineural ropivacaine attained this analgesia discharge criterion in a median $\left(25^{\text {th }}-75^{\text {th }}\right.$ percentiles $)$ of $20(0-38)$ hours, compared with 38 (15-64) hours for those of the control group (Estimated ratio $=0.52$, 95\% confidence interval: 0.28-0.84; p=0.009). "Eve": Evening; and "Nit": Night. 
Intravenous Opioid (Morphine)

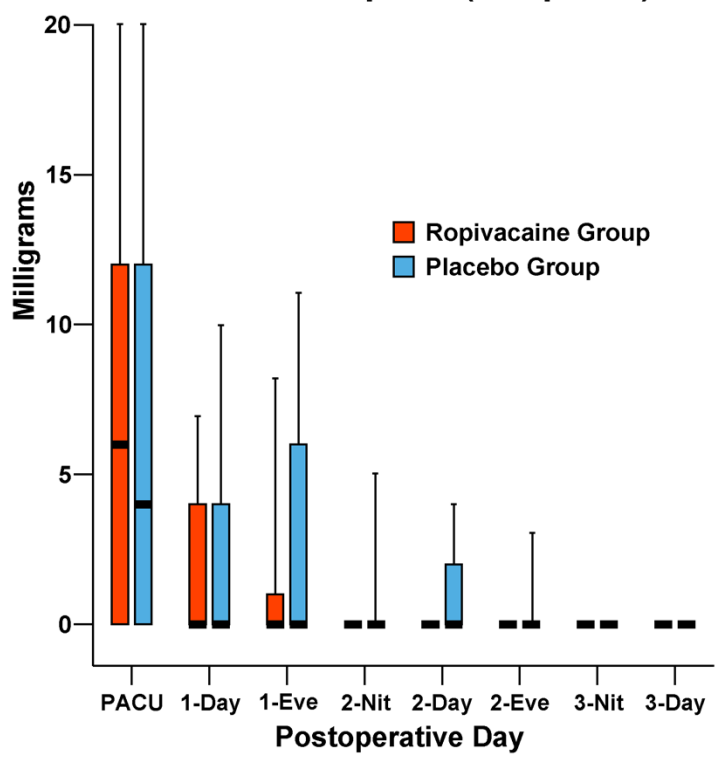

Perineural Infusion

$\square$ Placebo Group Ropiv Normal Saline

$\square$ Ropivacaine Group

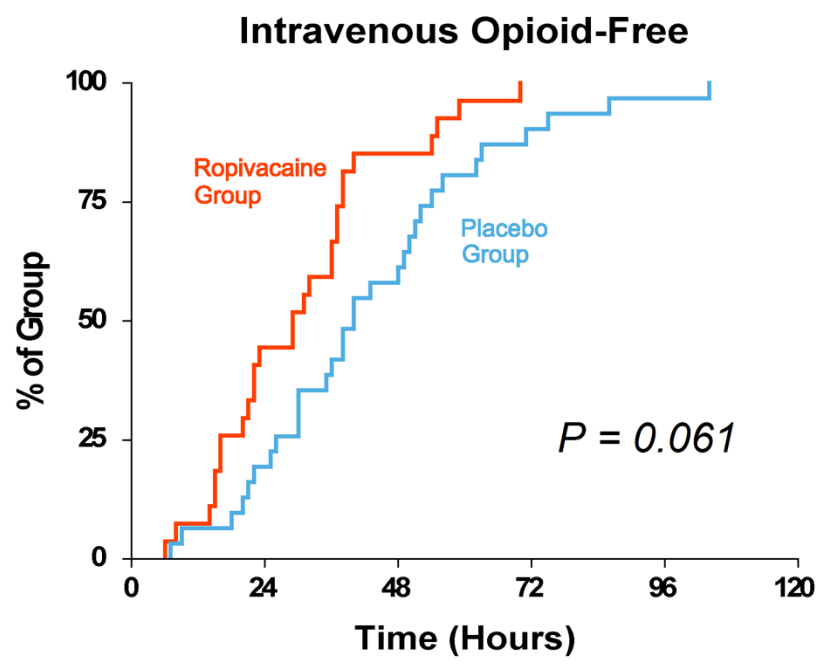

Figure 3.

Effects of femoral perineural ropivacaine infusion on intravenous morphine consumption following total knee arthroplasty. Data are expressed as median (horizontal bar) with $25^{\text {th }}$ $75^{\text {th }}$ (box) and $10^{\text {th }}-90^{\text {th }}$ (whiskers) percentiles for subjects randomly assigned to group Ropivacaine (perineural ropivacaine from surgery through postoperative day 4) or group Placebo (perineural ropivacaine from surgery through 06:00 postoperative day 1 followed by perineural normal saline through postoperative day 4). Because each comparison dilutes all other P-values, we restricted our analysis to 11 comparisons among secondary end points. Pvalues are presented where statistical comparisons were applied. Kaplan-Meier estimates include the cumulative percentages of subjects with the discharge criterion of independence 
from intravenous opioids during the 8-hour period and subsequent time points. Subjects given four days of perineural ropivacaine attained this discharge criterion of independence from intravenous opioids during an 8-hour period in a median $\left(25^{\text {th }}-75^{\text {th }}\right.$ percentiles $)$ of 21 (0-37) hours, compared with $33(11-50)$ hours for those of the control group (Estimated ratio $=0.69,95 \%$ confidence interval: $0.42-1.01 ; p=0.061$ ). "Eve": Evening; and "Nit": Night. 
Ambulation Distance
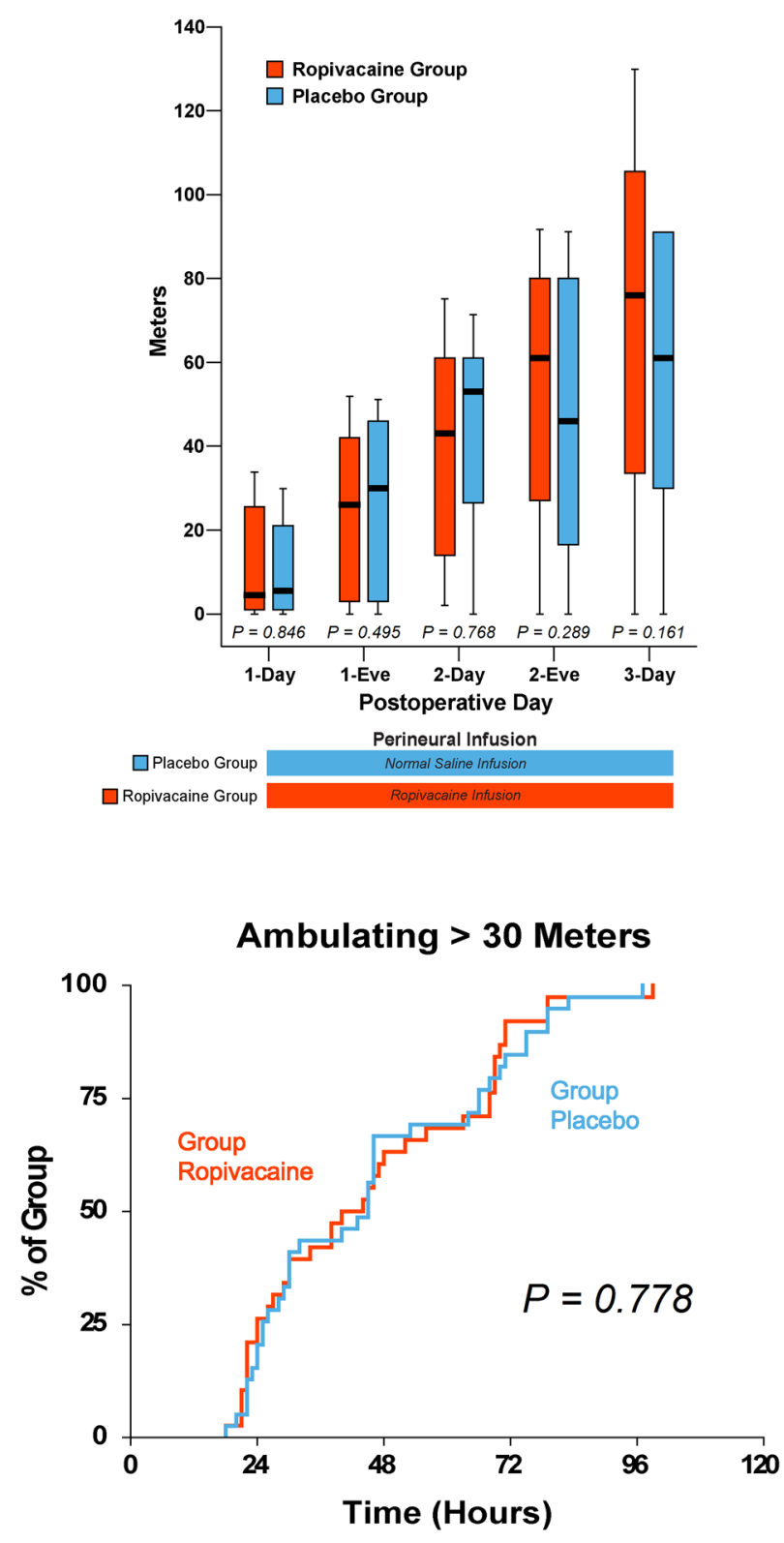

Figure 4.

Effects of femoral perineural ropivacaine infusion on ambulation following total knee arthroplasty. Data are expressed as median (horizontal bar) with $25^{\text {th }}-75^{\text {th }}$ (box) and $10^{\text {th }}$ $90^{\text {th }}$ (whiskers) percentiles for subjects randomly assigned to group Ropivacaine (perineural ropivacaine from surgery through postoperative day postoperative day 4) or group Placebo (perineural ropivacaine from surgery through 06:00 postoperative day 1 followed by perineural normal saline through postoperative day 4). Kaplan-Meier estimates include the cumulative percentages of subjects ambulating the discharge criterion of at least $30 \mathrm{~m}$ at each time point and subsequent time points. Subjects given four days of perineural ropivacaine attained this discharge criterion in a median $\left(25^{\text {th }}-75^{\text {th }}\right.$ percentiles) of 40 (2568 ) hours, compared with 45 (25-66) hours for those of the control group (Estimated ratio $=1.00,95 \%$ confidence interval: $0.75-1.26$; $p=0.778)$. "Eve": Evening. 


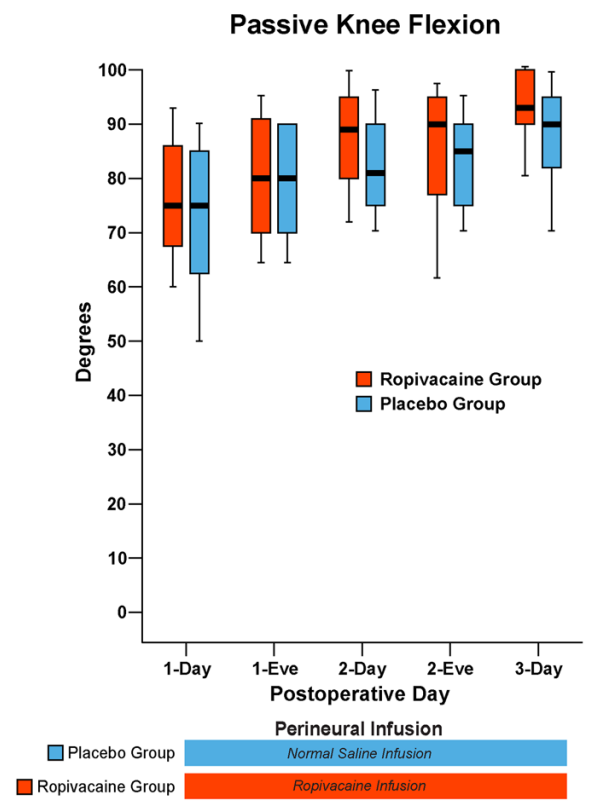

Passive Knee Extension

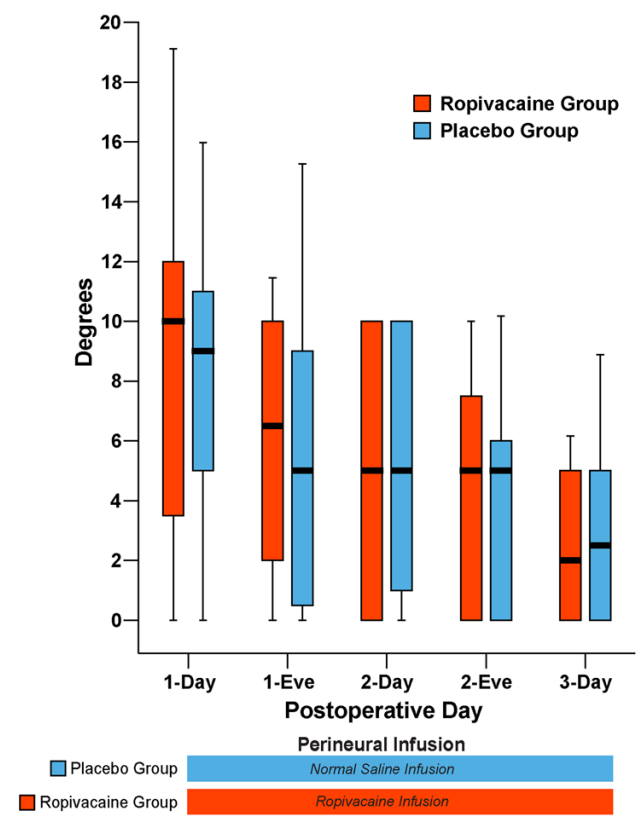

Figure 5.

Effects of femoral perineural ropivacaine infusion on passive knee flexion and extension following total knee arthroplasty. Data are expressed as median (horizontal bar) with $25^{\text {th }}$ $75^{\text {th }}$ (box) and $10^{\text {th }}-90^{\text {th }}$ (whiskers) percentiles for subjects randomly assigned to group Ropivacaine (perineural ropivacaine from surgery through postoperative day postoperative day 4) or group Placebo (perineural ropivacaine from surgery through 06:00 postoperative day 1 followed by perineural normal saline through postoperative day 4). Because each comparison dilutes all other P-values, we restricted our analysis to 11 comparisons among secondary end points. Therefore, statistics were not applied to the data of this figure. "Eve": Evening. 
Table 1

Pre-Randomization Subject Information

\begin{tabular}{lcc}
\hline & Ropivacaine Group (n=39) & Placebo Group (n=38) \\
\hline Age $(\mathrm{yr})$ & $61(58-67)$ & $66(60-70)$ \\
Sex (female/male) & $26 / 13$ & $25 / 13$ \\
Height $(\mathrm{cm})$ & $168(160-175)$ & $163(155-168)$ \\
Weight $(\mathrm{kg})$ & $82(69-95)$ & $81(70-88)$ \\
Body mass index $\left(\mathrm{kg} / \mathrm{m}^{2}\right)$ & $28(26-32)$ & $29(26-34)$ \\
ASA Physical Status & $2(2-2)$ & $2(2-2)$ \\
Minimum needle current $(\mathrm{mA})$ & $0.40(0.30-0.48)$ & $0.36(0.30-0.47)$ \\
Minimum catheter current $(\mathrm{mA})$ & $0.26(0.10-0.41)$ & $0.26(0.17-0.40)$ \\
Intraoperative fentanyl $(\mu \mathrm{gg})$ & $250(200-350)$ & $250(175-300)$ \\
Intraoperative morphine $(\mathrm{mg})$ & $0(0-8)$ & $0(0-6)$ \\
Catheter insertion to randomization $(\mathrm{h})$ & $23(21-23)$ & $22(20-23)$ \\
Surgery duration (min) & $138(114-155)$ & $133(111-156)$ \\
Tourniquet duration $(\mathrm{min})$ & $95(74-110)$ & $92(75-107)$ \\
\hline
\end{tabular}

Values are reported as median $\left(25^{\text {th }}-75^{\text {th }}\right.$ percentiles $)$

ASA: American Society of Anesthesiologists 


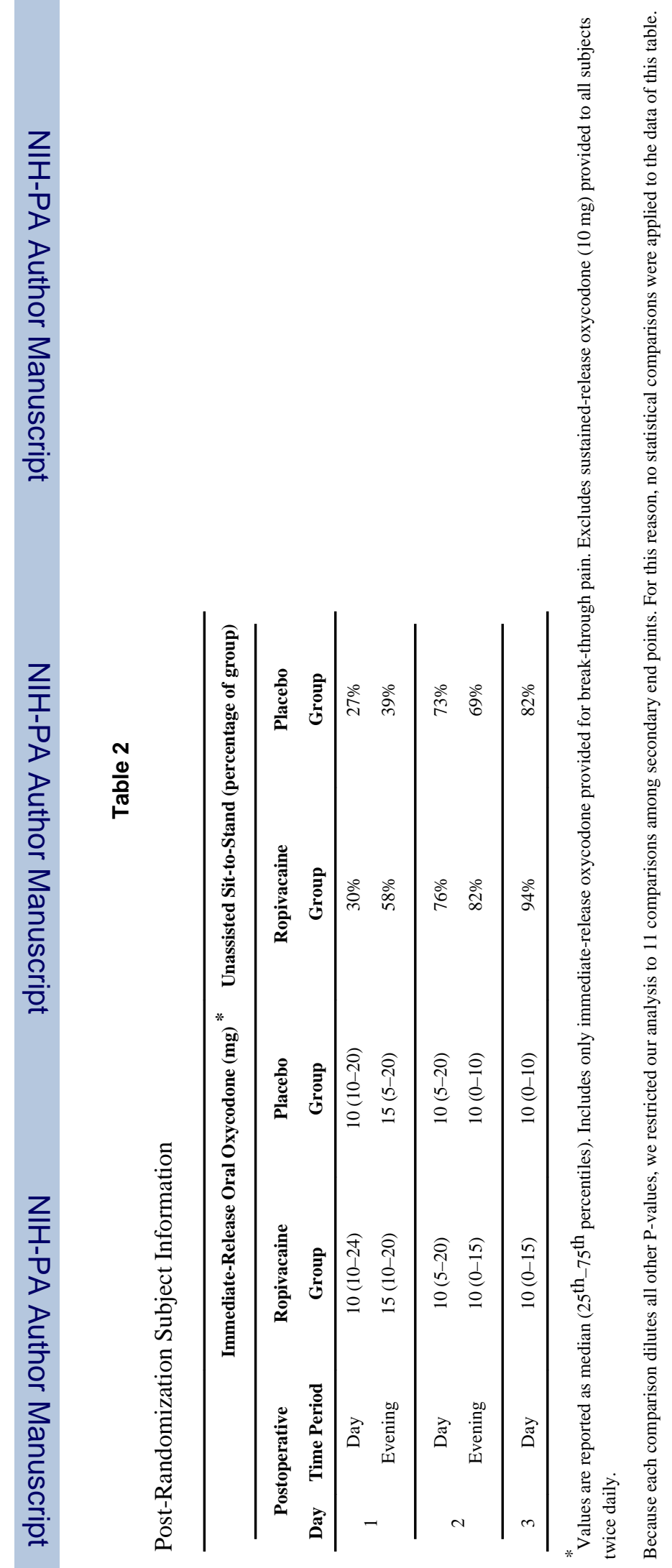

